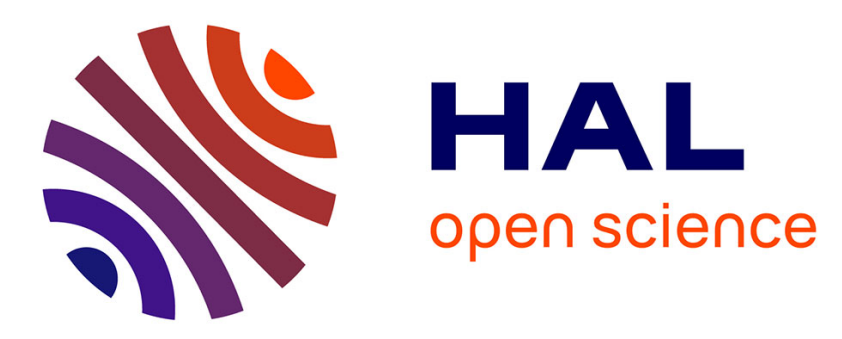

\title{
Détermination des paramètres d'une raie spectrale d'absorption soumise à des effets de pression
}

\author{
R. Occelli, G. Bachet, J.M. Moynault
}

\section{To cite this version:}

R. Occelli, G. Bachet, J.M. Moynault. Détermination des paramètres d'une raie spectrale d'absorption soumise à des effets de pression. Journal de Physique Lettres, 1980, 41 (22), pp.527-530. 10.1051/jphyslet:019800041022052700 . jpa-00231838

\section{HAL Id: jpa-00231838 https://hal.science/jpa-00231838}

Submitted on 1 Jan 1980

HAL is a multi-disciplinary open access archive for the deposit and dissemination of scientific research documents, whether they are published or not. The documents may come from teaching and research institutions in France or abroad, or from public or private research centers.
L'archive ouverte pluridisciplinaire HAL, est destinée au dépôt et à la diffusion de documents scientifiques de niveau recherche, publiés ou non, émanant des établissements d'enseignement et de recherche français ou étrangers, des laboratoires publics ou privés. 


\title{
Détermination des paramètres d'une raie spectrale d'absorption soumise à des effets de pression
}

\author{
R. Occelli, G. Bachet et J. M. Moynault \\ Laboratoire de Physique des Interactions Atomiques et Moléculaires, \\ Faculté des Sciences et Techniques de Saint-Jérôme, 13397 Marseille Cedex 4, France \\ (Reçu le 28 mai 1980, révisé le 18 septembre, accepté le 2 octobre 1980)
}

\begin{abstract}
Résumé. - Nous montrons par simulation qu'il est possible de déterminer avec précision, les paramètres d'un profil spectral perturbé par des effets de pression (déplacement, élargissement) en étudiant, à fréquence fixe, la variation d'absorption en fonction de la pression. Les résultats sont excellents même si les données sont affectées de fluctuations aléatoires.
\end{abstract}

\begin{abstract}
We show that it is possible to determine parameters of a spectral profile perturbed by pressure effects (shift and broadening) from the study of absorption versus pressure. Frequency is fixed. Simulation methods are used. The results are excellent in spite of the introduction of random fluctuations.
\end{abstract}

Dans une précédente publication [1], l'un d'entre nous a montré qu'il était théoriquement possible, à partir de la variation en fonction de la pression de l'absorption en un seul point du spectre, de déterminer avec précision l'intensité et la demi-largeur d'un profil Lorentzien.

Nous généralisons cette méthode et considérons le cas où le profil n'est pas seulement élargi par effet de pression, mais aussi déplacé $[2,3]$. Ce sont donc trois paramètres et non plus deux qu'il faut déterminer. De plus, nous analysons l'influence des fluctuations aléatoires sur les résultats de cette méthode.

Nous avons montré [4] qu'un profil Lorentzien reste valable au moins jusqu'à 40 bars dans le cas d'un perturbateur sphérique.

Nous nous plaçons dans le cas d'une raie isolée et en l'absence d'absorption induite décelable dans le perturbateur (exemple de HF perturbé par l'argon).

La détermination des paramètres du profil d'une raie à partir de la seule mesure de l'absorption $A_{v}(p)$ en fonction de la pression à fréquence fixe est d'un grand intérêt, elle devrait permettre une notable simplification de l'étude expérimentale des spectres IRL.

1. La méthode de calcul. - La méthode utilisée fait appel aux techniques numériques sur ordinateur. Elle peut se résumer ainsi :

- Dans une première étape, après avoir choisi un modèle théorique pour le profil de raie, nous constituons nos données en échantillonnant la fonction
$A_{v}(p)$ pour des valeurs de pression s'échelonnant de 1 à 20 bars.

- Dans une seconde étape, les valeurs précédentes tiennent le rôle de résultats expérimentaux et sont traitées par un programme de régression non linéaire $[5,6]$ qui permet, une fois le profil théorique fourni, la détermination précise des paramètres cherchés. On cherche alors à montrer qu'on peut ainsi retrouver les valeurs des paramètres qui nous ont permis d'élaborer les données.

Pour vérifier cette restitution, nous avons utilisé successivement :

a) La forme vraie de Lorentz (L) en

$$
1-\exp \left[-\frac{s l}{\pi \gamma} /\left[\left(\frac{v-v_{0}+\Delta v}{\gamma}\right)^{2}+1\right]\right]
$$

admise en proche infrarouge et dans laquelle :

$S \quad$ est l'intensité de la raie d'absorption;

$l$ est la longueur de la cuve;

$\Delta v$ le déplacement de la raie [5];

$\gamma \quad$ la demi-largeur de raie ;

$v_{0} \quad$ la fréquence correspondant au maximum de la raie;

$v \quad$ la fréquence d'observation;

dans la suite :

$S \quad$ est en $\mathrm{cm}^{-2}$;

$\gamma \quad$ en $\mathrm{cm}^{-1}$

$\Delta v \quad$ en $\mathrm{cm}^{-1}$

$v_{0}=40 \mathrm{~cm}^{-1}$. 
b) La forme de Lorentz $\left(\mathrm{L}_{\mathrm{c}}\right)$ corrigée du facteur de population qui l'asymétrise, en

$$
1-\exp \left[-\frac{f(v)}{f\left(v_{0}\right)} \times \frac{S l}{\pi \gamma} /\left[\left(\frac{v-v_{0}+\Delta v}{\gamma}\right)^{2}+1\right]\right]
$$

où :

$$
f(v)=v\left(1-\exp \left(\frac{h c v}{k T}\right)\right) .
$$

Cette forme étant la mieux adaptée dans l'IRL [2].

Nous nous sommes placés dans le cas où le faisceau lumineux dont on étudie l'absorption est une raie laser. La fonction d'appareil est de largeur nulle.

Le gaz absorbant est étudié successivement :

$1^{\text {o }}$ A concentration constante : l'intensité de la raie dépend alors directement de la densité des molécules actives.

$2^{\circ}$ A nombre de molécules actives constant : l'intensité de la raie est alors inchangée quelle que soit la pression.

Les simulations ont été faites pour plusieurs valeurs de $v-v_{0}$ échelonnées entre 0,2 et $1,5 \mathrm{~cm}^{-1}$. Nous n'avons décelé aucune influence sur les valeurs obtenues des paramètres recalculés. Les résultats présentés se rapportent à la valeur $0,5 \mathrm{~cm}^{-1}$.

De plus, étant donné que les relevés expérimentaux sont inévitablement entachés d'un bruit de fond, nous avons affecté les valeurs de l'absorption obtenues par échantillonnage, d'une erreur aléatoire, variable gaussienne de moyenne nulle et dont l'écart-type a été choisi de l'ordre de grandeur de l'incertitude communément admise expérimentalement pour ce type de mesure.

2. Etude à concentration constante de gaz actif. Dans le cas d'une concentration constante et dans le domaine étudié nous admettons que l'intensité $S$ de la raie, sa demi-largeur $\gamma$ et son déplacement $\Delta v$ sont des fonctions linéaires de la pression. Dans nos formules nous aurons donc :

$$
S=S_{1} \cdot p, \quad \gamma=\gamma_{1} p, \quad \Delta v=\Delta v_{1} p
$$

$S_{1}, \gamma_{1}, \Delta v_{1}$ représentant les paramètres rapportés à la pression unité.

Nous avons, bien entendu, admis que le perturbateur se comportait comme un gaz parfait, la pression étant alors proportionnelle à la densité.

Les valeurs initiales adoptées sont :

$$
\begin{aligned}
\frac{S l}{\pi} & =0,08 \mathrm{~cm}^{-1} ; \\
\gamma_{1} & =0,07 \mathrm{~atm}^{-1} \cdot \mathrm{cm}^{-1} ; \\
\Delta v_{1} & =0,02 \mathrm{~atm}^{-1} \cdot \mathrm{cm}^{-1}
\end{aligned}
$$

de l'ordre de grandeur de celles mesurées au laboratoire [7].

Le tableau I rassemble les résultats des calculs.
Tableau I. - Valeurs recalculées des paramètres, dans le cas d'une concentration constante, avec ou sans bruit de fond, pour les formes vraie $(\mathrm{L})$ et corrigée $\left(\mathrm{L}_{\mathrm{c}}\right)$ de Lorentz.

[Computed parameters. Constant concentration.]

$$
\begin{array}{ll}
\text { Cas expérimental } & \\
\text { en l'absence } & \text { avec bruit } \\
\text { de bruit de fond } & \text { de fond }
\end{array}
$$$$
\begin{array}{ccccc}
\text { Paramètre } & \mathrm{L} & \mathrm{L}_{\mathrm{c}} & \mathrm{L} & \mathrm{L}_{\mathrm{c}} \\
\hline & - & - & - & -
\end{array}
$$

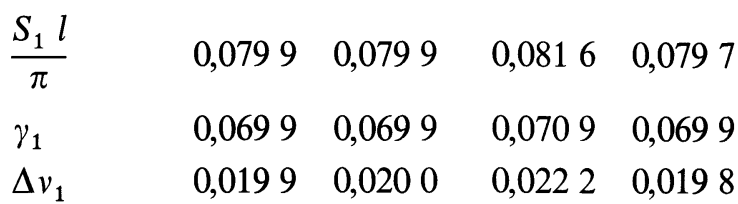

Ceux-ci ont été effectués avec les deux formes de raie, en envisageant, à chaque fois, l'absence et la présence de bruit parasite. On constate, pour tous les essais, une concordance parfaite très peu altérée par l'introduction d'incertitudes de mesure.

A titre d'exemple, la figure 1, représente la courbe d'absorption $A$ recalculée et les points expérimentaux, sans bruit de fond, dans le cas de la forme de Lorentz corrigée.

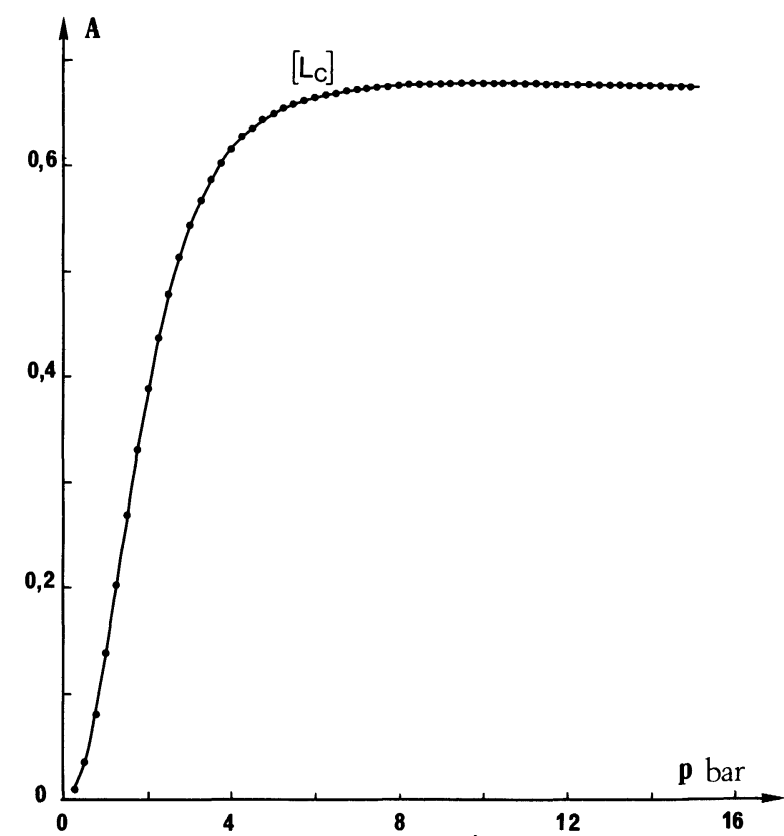

Fig. 1. - Points expérimentaux et courbe recalculée dans le cas d'une concentration constante, pour la forme corrigée de Lorentz et sans bruit de fond.

[Experimental points and computed curves. Constant concentration corrected Lorentz form without noise.]

Sur la figure 2 sont rapportées les courbes pour les deux formes de Lorentz avec bruit de fond.

3. Etude à nombre constant de molécules actives. Dans ce cas, on admet que l'intensité $S$ de la raie demeure inchangée lorsque la pression augmente. 


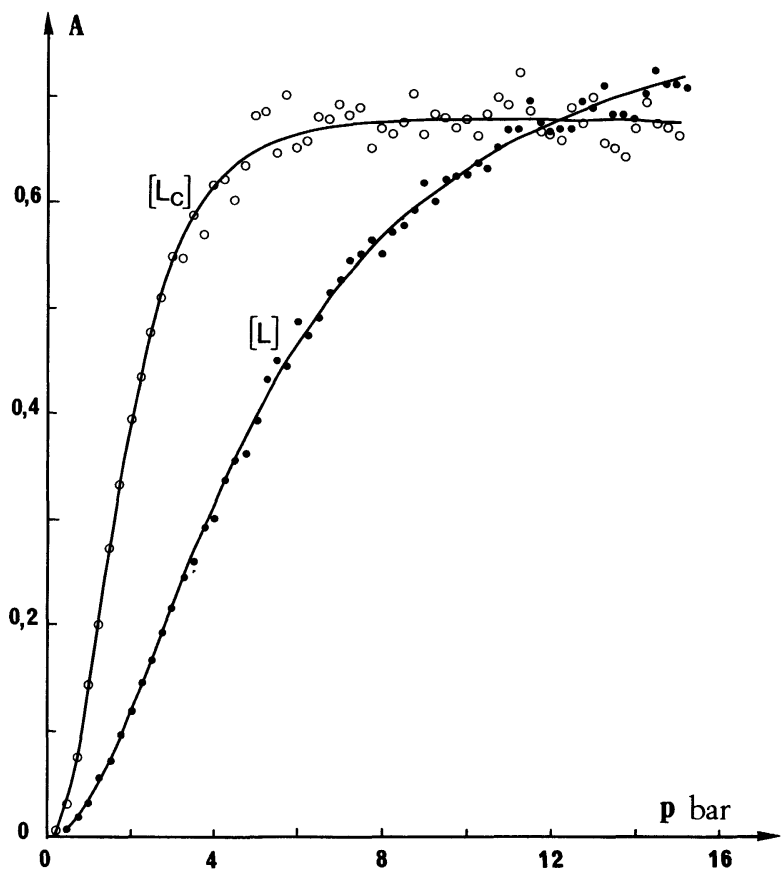

Fig. 2. - Points expérimentaux et courbe recalculée dans le cas d'une concentration constante pour les deux formes de Lorentz, avec bruit de fond.

[Experimental points and computed curves. Constant concentration. True and corrected Lorentz forms with noise.]

L'expression du profil de la raie devient alors :

$$
1-\exp \left[-\frac{S l}{\pi \gamma_{1} p} /\left[\left(\frac{v-v_{0}+\Delta v_{1} \cdot p}{\gamma_{1} p}\right)^{2}+1\right]\right] \text {. }
$$

Les valeurs de départ sont les mêmes que précédemment et les valeurs recalculées par le programme de régression non linéaire sont rassemblées dans le tableau II.

Tableau II. - Valeurs recalculées des paramètres pour un nombre de molécules actives constant avec et sans bruit de fond pour les deux formes de Lorentz.

[Computed parameters. Constant number of active molecules.]

\section{Cas expérimental}

en l'absence de bruit de fond

$\begin{array}{ccccc}\text { Paramètre } & \mathrm{L} & \mathrm{L}_{\mathrm{c}} & \mathrm{L} & \mathrm{L}_{\mathrm{c}} \\ - & - & - & - & - \\ \frac{S_{1} l}{\pi} & 0,0799 & 0,0799 & 0,0782 & 0,0799 \\ \gamma_{1} & 0,0700 & 0,0699 & 0,0705 & 0,0699 \\ \Delta v_{1} & 0,0199 & 0,0199 & 0,0193 & 0,0199\end{array}$

La figure 3 montre les points expérimentaux et la courbe lissée dans le cas de la forme de Lorentz corrigée, sans bruit de fond.

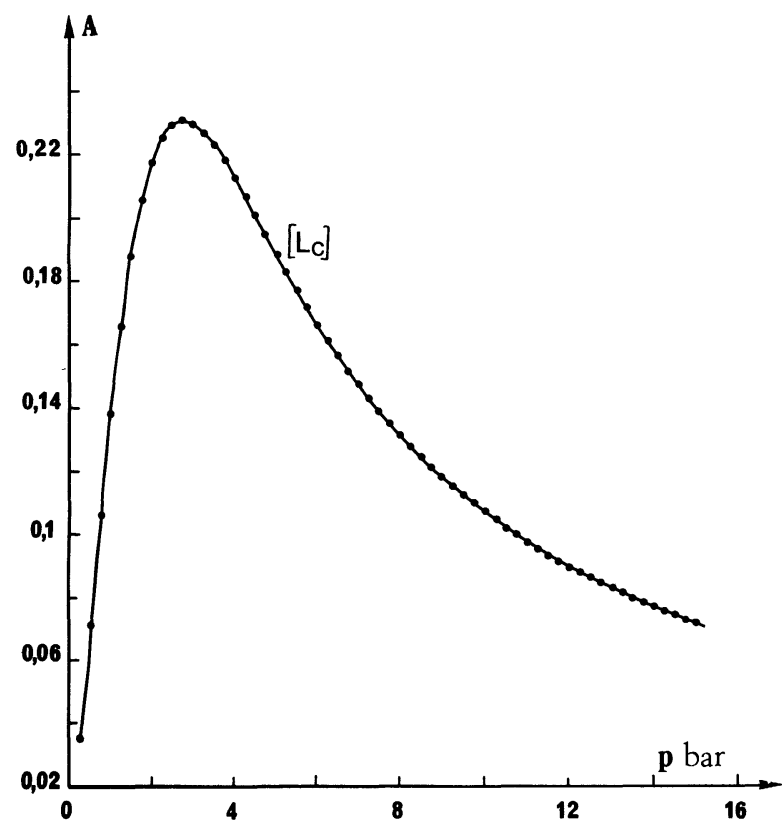

Fig. 3. - Même chose qu'en figure 1. Cas d'un nombre constant de molécules actives.

[Same as figure 1. Constant number of active molecules.]

La figure 4 permet de comparer les courbes obtenues avec bruit de fond, dans les cas des deux formes de Lorentz.

Là encore on remarque une bonne concordance entre valeurs initiales et valeurs recalculées. Elle est quasi parfaite pour la forme vraie de Lorentz.

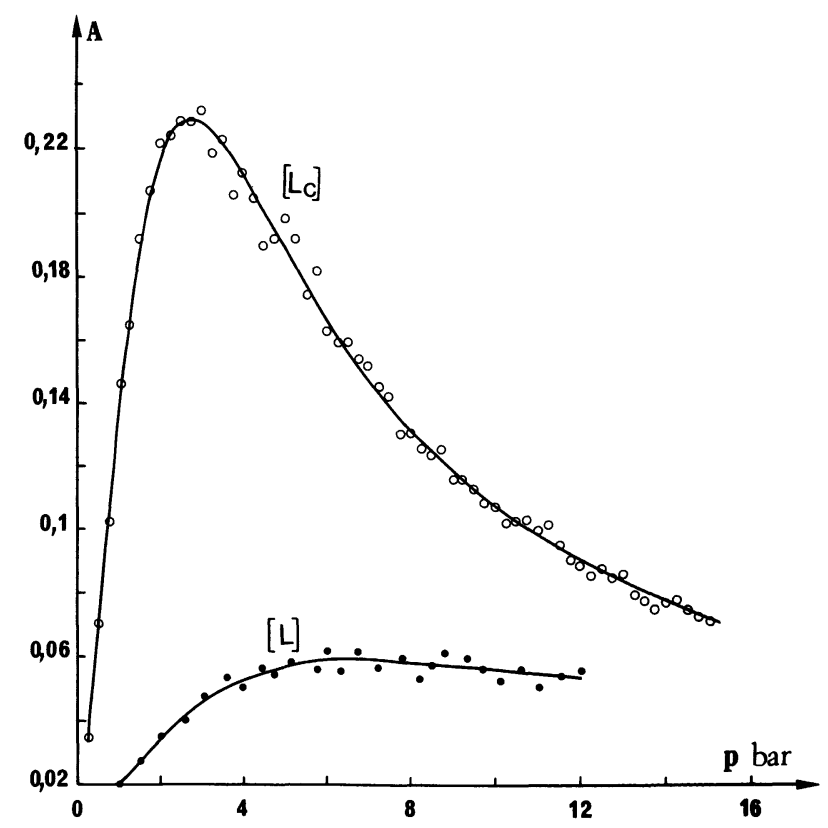

Fig. 4. - Même chose qu'en figure 2. Cas d'un nombre constant de molécules actives.

[Same as figure 2. Constant number of active molecules.]

4. Conclusion. - Nous avons ainsi montré par simulation numérique qu'il est possible à partir des 
résultats expérimentaux fournissant, à une fréquence donnée, l'absorption en fonction de la pression, de déterminer les trois paramètres fondamentaux : intensité, demi-largeur et déplacement qui caractérisent le profil d'une raie d'absorption, et ceci avec une grande précision, même en présence d'erreurs aléatoires entachant inévitablement les mesures expérimentales.

Cette technique doit avoir une application immé- diate à l'étude expérimentale des spectres IRL [7] où elle est facilitée par l'existence, dans ce domaine spectral, de nombreuses raies laser.

Nous envisageons ultérieurement la mise en œuvre expérimentale de cette méthode par comparaison des profils réels avec ceux calculés à l'aide des paramètres déduits à partir d'une série de mesures expérimentales de l'absorption en fonction de la pression à une fréquence donnée.

\section{Bibliographie}

[1] Bachet, G., C. R. Hebd. Séan. Acad. Sci. 280B (1975) 373.

[2] Bachet, G. et Coulon, R., C. R. Hebd. Séan. Acad. Sci. 284B (1977) 441.

[3] Boulet, C., Robert, D. et Galatry, L., J. Chem. Phys. 65, 12 (1976) 5302.
[4] Moynault, J. M., Bachet, G., Occelli, R., Coulon, R., J. Physique Lett. (à paraître).

[5] Pourcin, J. et Romanetti, R., Infrared Phys. 13 (1973) 161.

[6] Romanetri, R., Thèse de Doctorat ès Sciences (1973).

[7] BaChet, G. et Coulon, R., Infrared Phys. 18 (1978) 585. 